\title{
Enhanced Flatbed Tow Truck Model for Stable and Safe Platooning in the Presences of Lags, Communication and Sensing Delays
}

\author{
Alan ALI $^{1}$, Gaëtan GARCIA ${ }^{2}$ and Philippe MARTINET ${ }^{1}$
}

\begin{abstract}
Many ideas have been proposed to reduce traffic congestion. Driving a platoon of vehicles with constant spacing seems to be a promising idea as it increases traffic density. But keeping constant inter-vehicle spacing requires very reliable communication. Another control policy is to drive the platoon with a time headway between vehicles. It is a robust and well known policy but large inter-vehicle distances in addition to weak stability (unity error gain) near low frequencies make it less practical. We have proposed in [1], [2] a modification of the Constant Time Headway policy (CTH). This modification largely reduces the inter-vehicle distances using only one information shared between all vehicles.

In this work, we propose an additional modification of our control law. This modification makes our control law similar, in form, to the classical constant spacing policy, but it requires to share only one information between the vehicles. This modification improves the stability of the platoon and removes the weak stability of the CTH near low frequencies. We prove the robustness of the control law in the presence of actuating lags, sensing and communication delays. This proof can also be used to prove the stability of the classical constant spacing policy in the presence of all previous delays, which makes our result more general than those established in the literature. Safety is also discussed and the maximum acceptable communication delay without losing safety is determined. Simulations have been done in many critical scenarios.
\end{abstract}

\section{INTRODUCTION}

Many ideas have been proposed to solve traffic congestion. Platooning using automated cars seems to be a promising idea. It increases traffic density and safety, while simultaneously decreasing fuel consumption and driver tiredness.

Constant spacing and variable spacing policies are the main control policies used to control the spacing between vehicles [7], [13]. Variable spacing usually doesn't require a lot of data from other vehicles. In addition, it can ensure string stability using on-board information only [5], but intervehicle distances vary with speed and can be very large [14], hence traffic density is low. The most common variable spacing policy is the CTH [14]. The weak stability near low frequencies in addition to the large spacing when using CTH make it less practical. It was concluded that for high capacity traffic, the constant spacing policy is necessary, at the price of inter-vehicle communication [15].

Using communication may cause instability due to transmission delays or data drops. In [4] the effect of communication delays on string stability has been studied. It has been

\footnotetext{
${ }^{1}$ A. ALI and P. MARTINET are with Institut de Recherche en Communications et Cybernétique de Nantes (IRCCYN), Ecole Centrale de Nantes $(\mathrm{ECN})$, Nantes, France

${ }^{2}$ G. GARCIA is with Ecole Centrale de Nantes (ECN), Nantes, France
}

proved that the platoon becomes unstable for any propagation delays in the communicated leader informations, but the maneuvers that cause the string to be unstable are not given. This result was concluded by calculating the gain of the communication error transfer function near low frequencies and getting infinite gain. Practically, this proof represents an overconstrained condition because the only scenario which causes this instability is when we apply continuous acceleration. An attempt to remove this unsuitability was proposed in [16] by synchronizing all the vehicles to update their controllers at the same time and using the same leader information. But the effects of clock jitter, which can be seen as nonnegligible delay and hence may cause instability according to the results in [4], was briefly mentioned. [8] proved string stability for the leader-predecessor and predecessor-successor framework, neglecting information delays between vehicles. The effect of losing communication is presented in [15]. It has been proved that string stability can be retained, with limited spacing error, by estimating the state of the leading vehicle during losses.

Other delays and lags may be introduced in the physical systems by actuators and sensors. These delays may also have significant effects on stability if they are not taken into account. Stability conditions for many control laws, in the presence of lags and delays, can be found in [8], [9], [11], [14], [17]. A detailed study of the effect of delays and lags when using the classical time headway policy for homogeneous and heterogeneous platoons is found in [7]. The results show that the time headway policy is more immune to sensing and communication delays and actuating lags, than the constant spacing policy. But the large spacings between vehicles make it less important.

In [1], [2] we have proposed a modification of the $\mathrm{CTH}$ policy, which largely reduces inter-vehicle distances, to become nearly equal to the desired distance. These works were applied to urban platoons in [3]. In lateral control, we used sliding mode control to ensure stability and robustness. Safety of the platoon when using the modified control law was briefly studied in [1].

In this paper, we continue our previous work. We concentrate on controlling identical cars on nearly flat highways. The objectives of this paper are to remove the weak stability of the CTH near low frequencies and to prove the robustness of the platoon in presence of actuating lags, sensing and even communication delays. These objectives are achieved by proposing an additional modification to our control law and then by finding the conditions of the stability and safety. This can be done by finding an upper limit to the propagated error. 


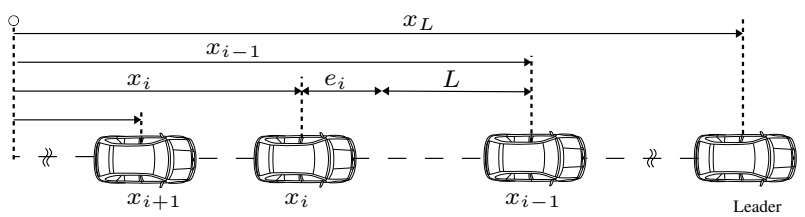

Fig. 1. A platoon

The stability of the platoon when using constant spacing policy is consequently obtained.

This paper is organized as follows: in section II we present a model for the vehicle, with and without taking the lags and delays into account, and a model for the platoon. The control law is developed in section III. String stability is proved in section IV. Safety is discussed in V. Then in section VI, we show simulation results. Discussions are done at VII. Conclusions and perspectives are done in the final section.

\section{MODELING}

\section{A. Longitudinal Model of the Vehicle}

Using Newton's law and taking into account the model of the engine, Then by applying the exact linearization we get a linear system [2], [7]:

$$
a_{i}(t)=\ddot{x}_{i}(t)=\dot{v}_{i}(t)=W_{i}(t)
$$

where $x_{i}, v_{i}, a_{i}$ are the position, speed and acceleration of the vehicle and $W_{i}$ is the control input.

\section{B. Vehicle model taking into account time delays and lags}

In reality, the ideal model given by (1) is not sufficient. Using it may lead to unstable control due to the presence of delays and lags in the real system.

Lags and time delays make the net engine torque not immediately equal to the desired torque calculated by the controller.

Another source of instability is the delay in the communicated data. This delay is due to transmission time, heavy communications or even data drops.

A vehicle model taking into account actuating lags and sensing delays is given in [7]. We extend this model to take into account communication delay so we get:

$$
\tau_{i} \ddot{v}_{i}(t)+\dot{v}_{i}(t)=W_{i}\left(t-\Delta_{i}, \tau_{c_{i}}\right)
$$

where $\tau_{i}$ is the combination of all the lags taken as a lumped lag, $\Delta_{i}$ is the combination of the all the time delays taken as a lumped delay, $\tau_{c_{i}}$ is the communication delay.

We also define $\Delta_{c_{i}}=\tau_{c_{i-1}}-\tau_{c_{i}}$ as the propagation delay between vehicle $i$ and vehicle $i-1$.

\section{Platoon Model}

The platoon is a set of vehicles moving together at the same speed and keeping a desired distance $L$ between consecutive vehicles.

The spacing error of the $i$-th vehicle, assuming a point mass model for all vehicles, is defined as follows:

$$
e_{i}=\Delta X_{i}-L \quad i=1 \ldots N
$$

Where $L$ is the desired inter-vehicle distance, $\Delta X_{i}=$ $x_{i-1}-x_{i}$ the spacing between vehicles $i$ and $(i-1)$, $\dot{e}_{i}=v_{i-1}-v_{i}$ the kinematic evolution of the spacing error and $N$ the total number of vehicles in the platoon.

The longitudinal model of the platoon, shown in fig. 2, is called flatbed tow track model [1]. It is a set of vehicles virtually connected by one-directional spring-damper systems, placed on a virtual truck which is set to drive at a speed $V$, the value of $V$ being known to all vehicles of the platoon. In this paper, we propose to add new virtual springs between each vehicle and the virtual truck. This enhances stability and makes our control law similar to the constant spacing policy. The main difference is that, in our case, the vehicles only receive the speed of the virtual truck $V$ and they calculate its position $X_{V}$ by integrating $V$, thus minimizing the amount of communicated data.

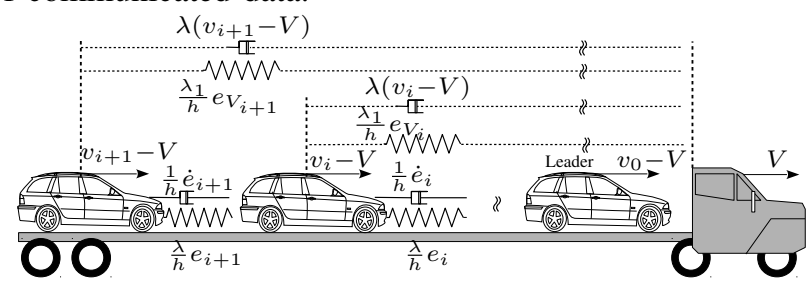

Fig. 2. Enhanced flatbed tow truck model

\section{CONTROL LAW AND SPACING ERROR DYNAMICS}

\section{A. Longitudinal Control}

Introducing the virtual truck in the new longitudinal model enables us to deal with relative speed instead of absolute speed, which enhances the performance of the longitudinal control by reducing the distance required to ensure string stability [1], [2]. This model is a modification of the classical time headway policy by subtracting a new term $V$ from all speeds. So the spacing error becomes [2]:

$$
\delta_{i}(t)=e_{i}(t)-h\left(v_{i}(t)-V(t)\right) \quad i=1 \ldots N
$$

where $h$ is the time headway, $V$ is a common speed value shared by all vehicles of the platoon, $\mathrm{V}$ must be the same value for all the vehicles at any sampling time [1], [2]. This value can be used to control the flow of the platoons.

We propose a modification to the control law we presented in [2] by adding a new term. The new term $e_{V_{i}}$ is proportional to the distance between the $i$-th vehicle and the virtual truck:

$$
W_{i}(t)=\frac{\dot{e}_{i}(t)+\lambda \delta_{i}(t)+\lambda_{1} e_{V_{i}(t)}}{h}, \quad i=1 \ldots N
$$

where $\lambda, \lambda_{1}$ are positive coefficients, $e_{V_{i}}=X_{V_{i}}-x_{i}-i L$, and $X_{V_{i}}$ is the position of the virtual truck, which can be calculated by integrating $V$.

\section{B. Longitudinal Control With Delays and Lags:}

When taking the delays and lags into account, the control law becomes the following:

$$
W_{i}=\frac{\dot{e}_{i}\left(t-\Delta_{i}\right)+\lambda \delta_{i}\left(t, \Delta_{i}, \tau_{c_{i}}\right)+\lambda_{1} e_{V_{i}}\left(t, \Delta_{i}, \tau_{c_{i}}\right)}{h}
$$




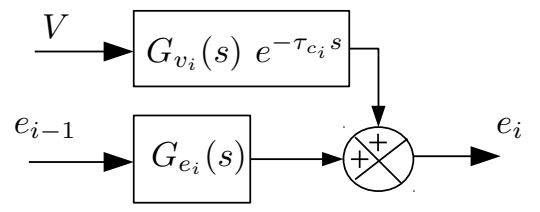

Fig. 3. Spacing error model taking into account delays and lags

where:

$\delta_{i}\left(t, \Delta_{i}, \tau_{c_{i}}\right)=e_{i}\left(t-\Delta_{i}\right)-h\left[v_{i}\left(t-\Delta_{i}\right)-V\left(t-\left(\Delta_{i}+\tau_{c_{i}}\right)\right)\right]$

$e_{V_{i}}\left(t, \Delta_{i}, \tau_{c_{i}}\right)=X_{V}\left(t-\left(\Delta_{i}+\tau_{c_{i}}\right)\right)-x_{i}\left(t-\Delta_{i}\right)-i L$

With no loss of generality, we assume that $v_{i}(0)=0$, $a_{i}(0)=0, e_{i}(0)=0$ for $0 \leq i \leq N$ at the initial conditions.

For an homogeneous platoon, we have: $\Delta_{i}=\Delta, \tau_{i}=\tau$, $\tau_{c_{i-1}}-\tau_{c_{i}}=\Delta_{c_{i}}=\Delta_{c}$ for $1 \leq i \leq N$. Hence, $G_{e_{i}}=G_{e}$, $G_{V_{i}}=G_{V}$ for $1 \leq i \leq N$.

Using (6), (7) and (2) and then calculating the Laplace transform taking into account previous assumptions, we get:

$$
E_{i}(s)=G_{e}(s) E_{i-1}(s)+G_{V}(s) e^{-\tau_{c_{i}} s} V(s), \quad i=2 \ldots N
$$

where $E_{i}(s), V(s)$ are the Laplace transform of $e_{i}(t), V(t)$ respectively, and

$$
\begin{gathered}
G_{e}=\frac{(s+\lambda) e^{-\Delta s}}{h \tau s^{3}+h s^{2}+\left((1+h \lambda) s+\lambda+\lambda_{1}\right) e^{-\Delta s}} \\
G_{V}=\frac{\left(\lambda h s+\lambda_{1}\right) e^{-\Delta s}\left(e^{-\Delta_{c} s}-1\right)}{s\left(h \tau s^{3}+h s^{2}+\left((1+h \lambda) s+\lambda+\lambda_{1}\right) e^{-\Delta s}\right)}
\end{gathered}
$$

Equation (9) shows that the error of the $i$-th vehicle is not just a function of $e_{i-1}$, but also a function of the shared speed $V(s)$ as shown in fig. 3. This is due to the presence of a communication delay.

It is very important to calculate the dynamics of $e_{1}$, because it has an important effect on the stability and the safety of the platoon and it is different from the dynamics of other vehicles. By using (6), (7) and (2) and by adding the terms $\left(\tau h \ddot{v}_{0}+h \dot{v}_{0}+\lambda h v_{0}+\lambda_{1} x_{0}\right)$ to the left and right side of (6) we get the dynamics of $e_{1}$ as a function of $V$ and of the speed of the leader $v_{0}$ :

$$
\begin{aligned}
& \tau h \dddot{e}_{1}(t)+h \ddot{e}_{1}(t)+(1+\lambda h) \dot{e}_{1}(t-\Delta)+\lambda e_{1}(t-\Delta)= \\
& \tau h \ddot{v}_{0}(t)+h \dot{v}_{0}(t)+\lambda h v_{0}(t-\Delta)-\lambda h V\left(t-\left(\Delta+\tau_{c_{1}}\right)\right) \\
& +\lambda_{1} x_{0}(t-\Delta)-\lambda_{1} X_{V}\left(t-\left(\Delta+\tau_{c_{1}}\right)\right)
\end{aligned}
$$

We calculate the Laplace transform:

$$
E_{1}(s)=F_{e} V_{0}(s)-F_{V} V(s)
$$

where $V_{0}(s)$ is the Laplace transform of $v_{0}(t)$, and

$$
\begin{gathered}
F_{e}=\frac{\tau h s^{3}+h s^{2}+\left(\lambda h s+\lambda_{1}\right) e^{-\Delta s}}{s\left(\tau h s^{3}+h s^{2}+\left((1+\lambda h) s+\lambda+\lambda_{1}\right) e^{-\Delta s}\right)} \\
F_{V}=\frac{\left(\lambda h s+\lambda_{1}\right) e^{-\left(\Delta+\Delta_{c}\right) s}}{s\left(\tau h s^{3}+h s^{2}+\left((1+\lambda h) s+\lambda+\lambda_{1}\right) e^{-\Delta s}\right)}
\end{gathered}
$$

\section{STABILITY}

\section{A. String Stability of Longitudinal Control}

The general string stability definition in the time domain is given in [12]. In essence, it means that all the spacing errors are bounded if the initial states are bounded.

In [10] we find a sufficient condition for string stability:

$$
\left\|e_{i}\right\|_{\infty} \leq\left\|e_{i-1}\right\|_{\infty}
$$

which means that the spacing error must not increase as it propagates through the platoon. To verify this condition, the spacing error propagation transfer function is calculated $G_{i}(s)=\frac{E_{i}(s)}{E_{i-1}(s)}$.

A sufficient condition for string stability in the frequency domain is derived:

$$
\left\|G_{i}(s)\right\|_{\infty} \leq 1 \quad \text { and } \quad g_{i}(t)>0 \quad i=1,2 . . N
$$

where $g_{i}(t)$ is the error propagation impulse response of the $i$-th vehicle.

In the following, we prove the stability of the platoon in two steps: first by finding stability conditions taking into account sensing time delays and lags, then adding a communication delay and checking stability.

\section{B. System Stability With Time Delay and Lags:}

In this first step, we neglect communication delays $\left(G_{V}(s)=0\right)$ and we get stability conditions. All the equations and the conditions established here will also be used when taking into account the communication delay. So we get:

$$
E_{i}(s)=G_{e}(s) E_{i-1}(s)
$$

In this case we can use (17) to check the stability, so we have to verify $\left\|G_{e}(\omega)\right\|_{\infty}<1$.

We have:

$$
\left\|G_{e}(\omega)\right\|=\sqrt{\frac{\mu_{0}}{\mu_{0}+\mu+\lambda_{1}^{2}+2 \lambda \lambda_{1}}}
$$

Where $\mu=\tau^{2} h^{2} \omega^{6}+\left[h^{2}-2 h \tau(1+\lambda h) \cos (\Delta \omega)\right] \omega^{4}-$ $2 h \sin (\Delta \omega)\left(1+\lambda(h-\tau)-\lambda_{1} \tau\right) \omega^{3}+[2 \lambda h(1-\cos (\Delta \omega))+$ $\left.\lambda^{2} h^{2}-2 \lambda_{1} h \cos (\Delta \omega)\right] \omega^{2}, \mu_{0}=\omega^{2}+\lambda^{2}$.

To ensure stability, it is sufficient to satisfy:

$$
\mu+\lambda_{1}^{2}+2 \lambda \lambda_{1} \geq 0
$$

A sufficient condition to satisfy (20) is to make $\mu \geq 0$.

$1-\cos (\Delta \omega) \geq 0$ is always true. We take $1+\lambda(h-\tau)-\lambda_{1} \tau \geq 0$ to make the coefficients of $\sin (\Delta \omega) \omega^{3}$ always negative and we have $\sin (\Delta \omega) \leq \Delta \omega$ for all $\omega>0$; this give us the following:

$$
\mu \geq \alpha_{1} \omega^{6}+\alpha_{2} \omega^{4}+\alpha_{3} \omega^{2}
$$

Where $\alpha_{1}=h^{2} \tau^{2}, \alpha_{3}=\lambda^{2} h^{2}-2 \lambda_{1} h, \alpha_{2}=\beta_{1} h^{2}+\beta_{2} h$, $\beta_{1}=(1-2 \lambda \tau-2 \lambda \Delta), \beta_{2}=2\left(\left(\lambda+\lambda_{1}\right) \tau \Delta-(\tau+\Delta)\right)$

If the following condition

$$
\alpha_{1} \omega^{6}+\alpha_{2} \omega^{4}+\alpha_{3} \omega^{2} \geq 0
$$

holds, then $\mu \geq 0$ is satisfied.

(22) is equivalent to the following inequality for all $\omega$ : 


$$
\alpha_{1} \omega^{4}+\alpha_{2} \omega^{2}+\alpha_{3} \geq 0
$$

A sufficient condition that makes the inequality hold is that all the coefficients $\alpha_{1}, \alpha_{2}, \alpha_{3}$ be positive.

We get a group of conditions that satisfy the stability of the platoon in the presence of lags and sensor delays:

$$
\left\{\begin{array}{l}
\lambda \leq \frac{h-2(\Delta+\tau)+2 \lambda_{1} \tau \Delta}{2(h(\Delta+\tau)-\Delta \tau)} \quad \text { and } \quad \frac{\lambda_{1}}{\lambda}<\frac{h}{2} \quad \text { and } \\
\lambda \geq \frac{\lambda_{1} \tau-1}{h-\tau} \quad \text { and } h \geq 2(\Delta+\tau)+2 \lambda_{1} \tau \Delta
\end{array}\right\}
$$

The last condition is to ensure that $\lambda \geq 0$.

\section{System Stability with Communication Delays:}

Stability can be verified easily using condition (17) when the error $e_{i}$ is a function of the previous error $e_{i-1}$ only. When the error becomes a function of additional variables we have to check the maximum limits of the spacing error. The system is stable if the spacing errors in the platoon are always bounded [12].

In the following we study the limits of the spacing error of the $i$-th vehicle when $N \longrightarrow \infty$.

Using (9), and omitting $(s)$ in $G_{e}(s), G_{V}(s), E_{1}(s)$ for simplicity, we can get the relation between $E_{i}(s)$ and $E_{1}(s)$ for $i=3, \ldots, N$ :

$$
\begin{aligned}
& E_{i}(s)=G_{e}^{i-1} E_{1}+G_{V} e^{-i \Delta_{c} s} \sum_{j=0}^{i-2} G_{e}^{j} e^{-j \Delta_{c} s} V(s) \\
& E_{i}(s)=G_{e}^{i-1} E_{1}+G_{V} e^{-i \Delta_{c}} \frac{1-\left(G_{e} e^{-\Delta_{c} s}\right)^{i-2}}{1-G_{e} e^{-\Delta_{c} s}} V(s)
\end{aligned}
$$

For $i=2$ the relation is given by :

$$
E_{2}(s)=G_{e} E_{1}+G_{V} e^{-\tau_{c_{2}} s} V(s)
$$

For any transfer function $G(s),\|G(\omega)\|_{\infty}=\|g(t)\|_{1}$ if its impulse function $g(t)$ is positive [12].

From (26) and the previous note we get:

$$
\left\|e_{i}(t)\right\|_{\infty} \leq \xi_{1}+\xi_{2}
$$

Where $\xi_{2}=\left\|G_{v}(\omega)\right\|_{\infty}\left\|\frac{1-\left(G_{e}(\omega) e^{-j \Delta_{c} \omega}\right)^{i-2}}{1-G_{e}(\omega) e^{-j \Delta_{c} \omega}}\right\|_{\infty}\|V(t)\|_{\infty}$, $\xi_{1}=\left\|G_{e}(\omega)\right\|_{\infty}^{i-1}\left\|e_{1}(t)\right\|_{\infty}$.

The first term $\xi_{1}$ is bounded (for all $\omega$ and $N \longrightarrow \infty$ ) if $\left\|G_{e}(\omega)\right\|_{\infty} \leq 1$ and $\left\|e_{1}(t)\right\|_{\infty}$ is bounded. For $N \longrightarrow$ $\infty$ these conditions make $\left\|G_{e}(\omega)\right\|_{\infty}^{i-1}\left\|e_{1}(t)\right\|_{\infty} \longrightarrow 0$ so the effect of $e_{1}$ disappears for the "infinity" vehicle. The conditions that keep $\left\|G_{e}(\omega)\right\|_{\infty} \leq 1$ are already given in (24). From (13) we can prove that $\left\|e_{1}(t)\right\|_{\infty}$ is also bounded because $\left\|F_{e}(\omega)\right\|$ and $\left\|F_{V}(\omega)\right\|$ converge toward zero for high frequencies. For low frequencies, $e_{1}$ becomes equal to $\lambda h\left(V-v_{i}\right)+\lambda_{1}\left(X_{V}-x_{0}\right)$. This can be bounded if we choose $V$ correctly. For all other frequencies, the denominator of $\left\|F_{e}\right\|$ and $\left\|F_{V}\right\|$ is always larger or equal to $\sqrt{\omega^{2}\left(\lambda+\lambda_{1}\right)^{2}}$ (we already proved that $\mu \geq 0$ ). This means that the denominators are greater than zero for all non zero $\omega$; So $\left\|F_{e}\right\|_{\infty}$ and $\left\|F_{V}\right\|_{\infty}$ are bounded for all non zero $\omega$, respectively by $\alpha_{1}, \alpha_{2}$. Then the first term always converge toward zero for all $\omega$ and when $N$ goes to infinity.

Concerning the second term $\xi_{2}$, from (41) we can prove $0<\left\|1-G_{e} e^{-\Delta_{c} s}\right\| \leq 2,0<\left\|1-\left(G_{e} e^{-\Delta_{c} s}\right)^{i}\right\| \leq 2$. We have also proved in (47) that $\left\|G_{V}\right\|$ is always limited for all $\omega$; so $\xi_{2}$ is limited for all frequencies even when $\omega=$ 0 , hence the platoon is stable for bounded communications delays. Clock jitter effect may be also taken in to account by this delay.

We can see that the platoon can be stable in the presence of lags, sensing and even communication delays. The stability conditions are given by (24) and in appendix in (40 and 45). These conditions can be applied to the constant spacing policy and we can get a stable platoon, contrary to the conclusion in [4]. We can also see that by adding the new term $\lambda_{1}$, the error transfer function $\left\|G_{e}(0)\right\|$ becomes always smaller than 1. This removes the weak stability in CTH law, this weak stability appear in CTH near low frequencies when the error transfer function gain becomes equal to 1 so the error is not damped. The maximum acceptable communication delay $\Delta_{c_{\max }}$ is defined by safety conditions.

\section{SAFETY}

To ensure safety of the platoon we have to ensure that the maximum error is smaller than the desired distance $\max \left(e_{i}(t)\right)<L$.

For the first car, we want to ensure that $\left|e_{1}(t)\right|$ is always less than $L$. By adding $\left(\tau h \ddot{V}+h \dot{V}+\lambda h V(t-\Delta)+\lambda_{1} x_{V}(t-\right.$ $\Delta)$ ) to the left and right side of (12), then by calculating the Laplace transform we get:

$$
E_{1}(s)=-K_{e}(s) E_{V}(s)+K_{V}(s) A_{V}(s)+G_{V}(s) V(s)
$$

where $E_{V}(s), A_{V}(s)$ are the Laplace transform of $e_{V}(t)=X_{v}-x_{0}$ and the acceleration of the virtual truck $a_{V}(t)$ respectively.

$$
\begin{aligned}
K_{e} & =\frac{\tau h s^{3}+h s^{2}+\left(\lambda h s+\lambda_{1}\right) e^{-\Delta s}}{\tau h s^{3}+h s^{2}+\left((1+\lambda h) s+\lambda+\lambda_{1}\right) e^{-\Delta s}} \\
K_{V} & =\frac{\lambda h s+h}{\tau h s^{3}+h s^{2}+\left((1+\lambda h) s+\lambda+\lambda_{1}\right) e^{-\Delta s}}
\end{aligned}
$$

If we choose the leader speed $v_{0}$ as the common shared speed $V$, the error $e_{v}$ becomes zero; so the first error becomes:

$$
E_{1}(s)=K_{V}(s) A_{V}(s)+G_{V}(s) V(s)
$$

So we have:

$\left\|e_{1}(t)\right\|_{\infty} \leq\left\|K_{V}\right\|_{\infty} \max \left|a_{V}(t)\right|+\left\|G_{V}\right\|_{\infty} \max |V(t)|$

To ensure that $e_{1} \leq L$, it is sufficient to verify:

$$
\left\|K_{V}\right\|_{\infty} \max \left|a_{V}(t)\right|+\left\|G_{V}\right\|_{\infty} \max |V(t)| \leq L
$$

From (50) and (34) we get the safety condition for first error:

$$
\Delta_{c} \leq\left(L-\frac{h}{\lambda+\lambda_{1}} \max \left|a_{V}(t)\right|\right) \frac{\lambda+\lambda_{1}}{\lambda_{1} \max |V(t)|}
$$


For other errors, from (9) and for the transfer functions $G_{e_{i}}(s), G_{V_{i}}(s)$ with positive impulse functions $g_{e_{i}}(t), g_{V_{i}}(t)$ we get for $i=2 \ldots N$ :

$$
\left\|e_{i}(t)\right\|_{\infty} \leq\left\|G_{e_{i}}(\omega)\right\|_{\infty}\left\|e_{i-1}(t)\right\|_{\infty}+\left\|G_{V_{i}}(\omega)\right\|_{\infty}\|V(t)\|_{\infty}
$$

In this case, the following condition is a sufficient condition for safety $\left\|G_{e_{i}}(\omega)\right\|_{\infty} L+\left\|G_{V_{i}}(\omega)\right\|_{\infty} \max (|V(t)|) \leq L$ then $\left\|G_{V_{i}}(\omega)\right\|_{\infty} \leq\left(1-\left\|G_{e_{i}}(\omega)\right\|_{\infty}\right) L / \max |V(t)|$

Then by using (47) and (41) we get the upper limit for communication propagation delay that prevents collisions:

$$
\Delta_{c} \leq \frac{L}{\max |V(t)|}
$$

\section{SIMULATIONS}

Simulations have been done using Matlab. A large platoon, of 60 vehicles, is simulated. In practice, the platoon would likely be much more smaller. Here we use this large platoon just to check that the error does not increase, even for a vehicle with a large index. We tested the system in critical scenarios (acceleration from zero to maximum speed with maximum acceleration, moving at maximum speed and then applying emergency stop from maximum speed) to check the validity of stability and safety conditions at their limits. The desired inter-vehicle distance is $L=12 \mathrm{~m}$. The maximum speed of the leader is $140 \mathrm{~km} / \mathrm{h}$. We take the maximum acceleration equal to $5 \mathrm{~m} . \mathrm{s}^{-2}$ which is beyond the comfortable acceleration and also exceeds the ability of most vehicles. According to [10] the lag delay is about $70 \mathrm{~ms}$, we take larger values for the actuating lags equal to $\tau=200 \mathrm{~ms}$ and a sensing delay equal to $\Delta=200 \mathrm{~ms}$. According to [6] the communication propagation delay is smaller than $\Delta_{c}=50 \mathrm{~ms}$. We take $h=2, \lambda=0.7, \lambda_{1}=0.2$. For clarity, we only show the speed of one of ten vehicles.

We can see in fig. 4 that the platoon is stable, since the errors do not increase through the platoon. In addition, we can see that the spacings between vehicles are always larger than zero, so the platoon is safe. Previously in [2] we chose $l=5 \mathrm{~m}$, we notice here that we have enlarged the desired inter-vehicle distance to compensate for the errors generated by lags and delays. In practice, we would add an additional safety margin distance in the desired distance to ensure more safety.

\section{DISCUSSION}

- The new modification improves the performance of our control law, without requiring new data from other vehicles. Each car can calculate the current position of the truck using the shared speed $V$. So $X_{V}$ is always the same for all vehicles.

- In the event of a loss of communication, all vehicles switch to stable autonomous mode (classical time headway) by making $V$ and $X_{V}$ smoothly become equal to 0 and $x_{i}$ (for the $i$-th vehicle) respectively.

- Losing communication prevents the vehicles from having the same value for $X_{V}$; so it is necessary to update
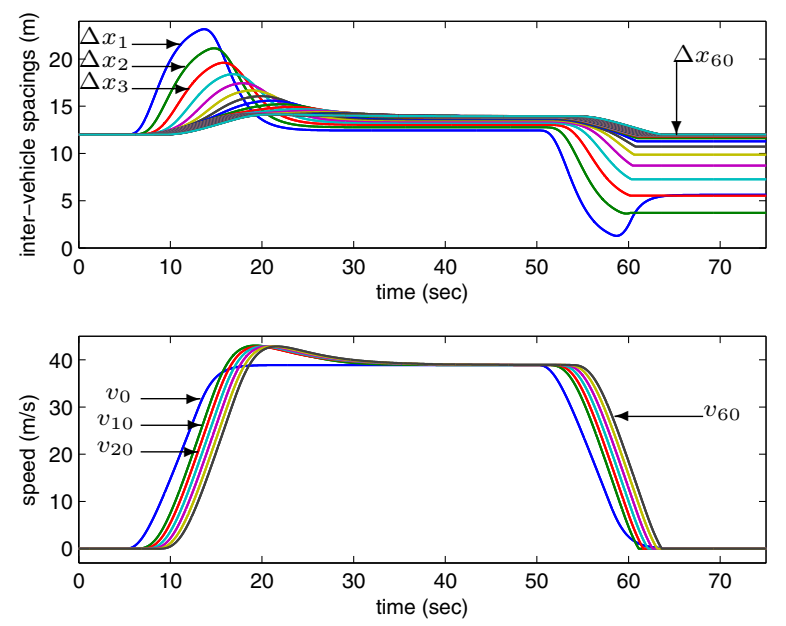

Fig. 4. Inter-vehicle spacings in the presence of lags, sensing and communication delays

$X_{V}$ for all the vehicles after each loss of communication in a common predetermined way.

- The integration of the error in $V$ will have no effect on stability because this error will be the same for all vehicles. This error will have an effect on safety between the first vehicle and the leader. As we saw before, the most important condition to maintain stability is to keep $X_{v}-x_{0}$ limited.

- We can see that the control law proposed in this paper, with the one proposed in [2] in addition to the classical time headway, represent an integrated framework for controlling the platoon. These laws enable us to switch from one law to another smoothly, without losing stability, according to decreasing communication capability.

\section{CONCLUSION}

In this paper, we have addressed the control of platoons on highways. The longitudinal dynamics are modeled using modified flatbed tow truck model. We proved the robustness of this control law to lags, delays and even communication delays. Sufficient stability conditions have been given in equations (24), (40) and (45). The safety of the platoon was also studied and corresponding sufficient conditions established in equations (37), (35), (40), (45) and (49). The maximum acceptable communication propagation delay without losing safety is bounded by the conditions given in equation (35) and (37). In future work, non homogeneous platoons with non-homogeneous delays will be studied. Passenger comfort will also be taken into account.

\section{APPENDIX}

- To ensure string stability and safety, we have to find $\left\|G_{e}\right\|_{\infty}$ and to make it as small as possible.

From (19) and when $\omega=0$ we have $\left\|G_{e}(0)\right\|=\frac{\lambda}{\lambda+\lambda_{1}}$. We also have, from (19) and (21): 


$$
\left\|G_{e}\right\| \leq \sqrt{\frac{\omega^{2}+\lambda^{2}}{\omega^{2}+\lambda^{2}+\alpha_{1} \omega^{6}+\alpha_{2} \omega^{4}+\alpha_{3} \omega^{2}+\lambda_{1}^{2}+2 \lambda \lambda_{1}}}
$$

We already found in (24) the conditions that make $\left\|G_{e}(\omega)\right\| \leq 1$. It is always better to make $\left\|G_{e}(\omega)\right\|_{\infty}$ as small as possible to decrease the amplitude of the propagated error. In our case we make $\left\|G_{e}(\omega)\right\|_{\infty}=\left\|G_{e}(0)\right\|<1$. This can be verified by imposing an upper limit for the right term of (38) for all $\omega$ :

$$
\sqrt{\frac{\omega^{2}+\lambda^{2}}{\omega^{2}+\lambda^{2}+\alpha_{1} \omega^{6}+\alpha_{2} \omega^{4}+\alpha_{3} \omega^{2}+\lambda_{1}^{2}+2 \lambda \lambda_{1}}} \leq\left\|G_{e}(0)\right\|
$$

This imposes the following condition:

$$
\alpha_{3} \geq \frac{\lambda_{1}}{\lambda}-1
$$

So:

$$
\left\|G_{e}(\omega)\right\|_{\infty}=\left\|G_{e}(0)\right\|=\frac{\lambda}{\lambda+\lambda_{1}}<1
$$

- We also want to find $\left\|G_{V}\right\|_{\infty}$. We have:

$$
\left\|G_{V}(\omega)\right\|=\sqrt{\frac{\left(\lambda^{2} h^{2} \omega^{2}+\lambda_{1}^{2}\right)(2-2 \cos (\Delta c \omega))}{\omega^{2}\left(\mu_{0}+\mu+\lambda_{1}^{2}+2 \lambda \lambda_{1}\right)}}
$$

Using Taylor series near $\omega=0$ we can find that $\sqrt{2-2 \cos \left(\Delta_{c} \omega\right)} \rightarrow \Delta_{c} \omega$. So we can find $\left\|G_{V}(0)\right\|$ :

$$
\left\|G_{V}(\omega)\right\| \stackrel{\omega \longrightarrow 0}{\longrightarrow} \frac{\lambda_{1}}{\lambda+\lambda_{1}} \Delta c
$$

Again, it is better to make $\left\|G_{V}\right\|_{\infty}$ as small as possible to decrease the error generated due to the communication delay, and propagated through the platoon.

So we want to make $\left\|G_{V}(\omega)\right\|_{\infty}=\left\|G_{V}(0)\right\|$. We can write $\left\|G_{V}\right\|$ as follows:

$$
\left\|G_{V}(\omega)\right\|=\underbrace{\sqrt{\frac{a_{1}}{a_{1}+b_{1}+\lambda_{1}^{2}+2 \lambda \lambda_{1}}}}_{\eta_{1}} \underbrace{\sqrt{\frac{(2-2 \cos (\Delta c \omega))}{\omega^{2}}}}_{\eta_{2}}
$$

where $b_{1}=\alpha_{1} \omega^{6}+\alpha_{2} \omega^{4}+\left(2 \lambda h\left(1-\cos \left(\Delta_{c} \omega\right)\right)+\right.$ $\left(1-2 \lambda_{1} h \cos \left(\Delta_{c} \omega\right)\right) \omega^{2}, a_{1}=\lambda^{2} h^{2} \omega^{2}+\lambda_{1}^{2}$.

$\eta_{1} \leq 1$ if $b_{1}+\lambda_{1}^{2}+2 \lambda \lambda_{1} \geq 0$. To satisfy this inequality, all the coefficients of $\omega$ must be positive, so:

$$
\lambda_{1} \leq \frac{1}{2 h}
$$

In addition, we always have $\eta_{2} \leq \Delta_{c}$ because:

$$
\eta_{2} \leq \sqrt{\frac{4 \sin ^{2}(\Delta c \omega / 2)}{\omega^{2}}} \leq \sqrt{\frac{4(\Delta c \omega / 2)^{2}}{\omega^{2}}} \leq \Delta c
$$

So $\left\|G_{V}(\omega)\right\| \leq\left\|G_{V}(0)\right\|$, for all $\omega$ :

$$
\left\|G_{V}(\omega)\right\|_{\infty}=\left\|G_{V}(0)\right\|=\frac{\lambda_{1}}{\lambda+\lambda_{1}} \Delta c
$$

- We calculate $\left\|K_{V}\right\|_{\infty}$ as follows:

$$
\left\|K_{V}\right\|=\sqrt{\frac{a_{2}}{a_{2}+b_{1}+\lambda_{1}^{2}+2 \lambda \lambda_{1}}}
$$

where $a_{2}=\tau^{2} h^{2} \omega^{2}+h^{2}$.

We have $\left\|K_{V}(0)\right\|=\frac{h}{\lambda+\lambda_{1}}$.

It is also better to make $\left\|K_{V}\right\|$ as small as possible so we try to make $\left\|K_{V}(\omega)\right\|_{\infty} \leq\left\|K_{V}(0)\right\|, \forall \omega$. This is satisfied under the following condition:

$$
\left(\lambda+\lambda_{1}\right)^{2} \tau^{2} \leq\left(\tau^{2}+\lambda^{2}\right) h^{2}-2 \lambda_{1} h
$$

So we get:

$$
\left\|K_{V}(\omega)\right\|_{\infty}=\left\|K_{V}(0)\right\|=\frac{h}{\lambda+\lambda_{1}}
$$

\section{REFERENCES}

[1] Ali, A.; Garcia, G. and Martinet, P., The flatbed platoon towing model for safe and dense platooning on highways, IEEE Intelligent Transportation systems Magazine, to be published, 2014.

[2] Ali, A.; Garcia, G. and Martinet, P., Minimizing the inter-vehicle distances of the time headway policy for platoons control in highways, 10th International Conference on Informatics in Control, Automation and Robotics (ICINCO13), pp. 417-424. SciTePress, Reykjavik, Iceland, July 29-31, 2013.

[3] Ali, A.; Garcia, G. and Martinet, P., Minimizing the inter-vehicle distances of the time headway policy for urban platoon control with decoupled longitudinal and lateral control, 16th International IEEE Conference on Intelligent Transportation Systems - (ITSC), pp. 18051810, The Hague, The Netherlands, 6-9 Oct. 2013.

[4] Hedrick, J. K.; Chen, Y. and Mahal, S., Optimized Vehicle Control/Communication Interaction in an Automated Highway System, Institute of Transportation Studies, Research Reports, Working Papers, Institute of Transportation Studies, UC Berkeley.2001

[5] Ioannou P., Chien C., Autonomous intelligent cruise control, IEEE Transactions on Vehicular Technology, 42(4):657-672, 1993.

[6] Jawhar I., Mohamed N., Usmani H., An overview of inter-vehicular communication systems, protocols and middleware, Journal of Networks, vol.8, no.12, pp.2749-2761, 2013

[7] Lingyun, Xiao and Feng, Gao, Practical String Stability of Platoon of Adaptive Cruise Control Vehicles, IEEE Transactions on Intelligent Transportation Systems, vol.12, no.4, pp.1184,1194, Dec. 2011

[8] Ling-yun, Xiao and Feng, Gao, Effect of information delay on string stability of platoon of automated vehicles under typical information frameworks,Journal of Central South University of Technology, Vol.17, no.6, pp 1271-1278, Dec.2010

[9] Lingyun, Xiao; Darbha, S. and Feng, Gao, Stability of String of Adaptive Cruise Control Vehicles with Parasitic Delays and Lags, 11th International IEEE Conference on Intelligent Transportation Systems ITSC 2008, vol., no., pp.1101,1106, 12-15 Oct. 2008

[10] Rajamani, R., Vehicle dynamics and control, Springer science, ISBN 0387263969, 2006.

[11] Rajamani, R. and Shladover S., An experimental comparative study of autonomous and co-operative vehicle-follower control systems, Transp. Res. Part C, vol. 9, no. 1, pp. 15-31, Feb. 2001.

[12] Swaroop, D., String stability of interconnected systems: An application to platooning in automated highway systems. UC Berkeley: California Partners for Advanced Transit and Highways (PATH), 1997.

[13] Swaroop, D., Hedrick, J., Chien, c. C., and Ioannou, P. A comparision of spacing and headway control laws for automatically controlled vehicles1. Vehicle System Dynamics, 23(1):597-625, 1994.

[14] Swaroop, D. and Rajagopal, K., A review of constant time headway policy for automatic vehicle following. In Proceedings IEEE Intelligent Transportation Systems, pp. 65-69, 2001.

[15] Teo, R.; Stipanovic, D.M. and Tomlin, C.J., Decentralized Spacing Control of a String of Multiple Vehicles Over Lossy Datalinks, IEEE Transactions on Control Systems Technology, vol.18, no.2, pp.469,473, March 2010.

[16] Xiangheng, Liu; Goldsmith, A. and Mahal, S.S.; Hedrick, J.K., "Effects of communication delay on string stability in vehicle platoons," Intelligent Transportation Systems, 2001. Proceedings. 2001 IEEE, vol., no., pp.625,630, 2001

[17] Yanakiev, D. and Kanellakopoulos, Ioannis, Longitudinal control of automated CHVs with significant actuator delays, IEEE Transactions on Vehicular Technology, vol.50, no.5, pp.1289,1297, Sep 200 